\title{
Taking advantage of oxidation to characterize thiol-containing polymer chains by MALDI-TOF mass spectrometry
}

\author{
Cassandre Gaillot, ${ }^{1}$ Frédéric Delolme, ${ }^{2}$ Laura Fabre, ${ }^{1}$ Marie-Thérèse Charreyre, ${ }^{1}$ Catherine Ladavière, ${ }^{1 *}$ \\ Arnaud Favier ${ }^{1 *}$ \\ ${ }^{1}$ Univ Lyon, Université Lyon 1, INSA de Lyon, CNRS, Laboratoire Ingénierie des Matériaux Polymères, UMR5223, F- \\ 69621 Villeurbanne, France. \\ ${ }^{2}$ Univ Lyon, SFR BioSciences, Université Lyon 1, ENS de Lyon, Protein Science Facility, CNRS UMS3444, INSERM \\ US8, F-69007 Lyon, France.
}

\begin{abstract}
MALDI-TOF mass spectrometry analyses revealed the oxidation of thiol-containing polymer chain-ends during sample preparation using THF as solvent. In these conditions, the extent of oxidation was hardly reproducible, and led to various types of oxidized compounds. Preparing the samples at the last minute using commercial THF stabilized with an anti-oxidant led to more reproducible results, with the least oxidation. However, it is demonstrated herein that thiol oxidation can be advantageously taken into profit to further ascertain the presence of the thiol at the polymer chain-end. To force thiol oxidation we used THF without any anti-oxidant stabilizer, thus more prone to form peroxides. Thiol-containing polymer chains can thereby be indirectly evidenced by the formation of oxidation products such as chain-chain disulfide bonds and sulfonic acid chains-ends. More importantly, in these oxidizing conditions and in the negative mode, sulfonic acid-terminated polymer chains can be more sensitively detected than thiol ones (the low pKa of sulfonic acids facilitating their anionization in MALDI source). In conclusion, performing MALDITOF mass spectrometry analyses in oxidizing conditions, as complement to regular analyses, was found to be very useful for the chain-end identification of different thiol-containing polymer chains.
\end{abstract}

Matrix-assisted laser light desorption ionization - time of flight mass spectrometry (MALDI-TOF MS) is a powerful technique for the analysis of macromolecular ions. It has been applied to the characterization of biomolecules such as peptides $^{1-2}$, and proteins ${ }^{3}$ but also synthetic and natural polymers. ${ }^{4}$ With this technique, the extent of fragmentation is limited thanks to soft desorption and ionization processes, giving access to the identification of individual macromolecules (precise $\mathrm{m} / \mathrm{z}$ value), even within a mixture or a chain distribution. Consequently, MALDI-TOF MS has become a privileged tool for the characterization of synthetic polymers, especially their chain-ends.

For sample analysis inside of the vacuum chamber of the mass spectrometer, a common solvent is generally used to prepare an analyte/matrix solution which is then spotted and air-dried at the surface of the metal target. Possible alteration of the sample may result from this process. In particular, the generation of different oxidation side-products may significantly complicate data analysis. This is especially a concern when analyzing macromolecules containing sulfur and thiols, although MALDI-TOF MS is a technique of choice for their characterization that is often difficult by other techniques, e.g. NMR.

MALDI-TOF MS has been widely used to detect naturally occurring thiols and their modifications in proteins or peptides (cysteine residues), including their oxidized forms. ${ }^{3}$ In this context, oxidation during sample preparation should be avoided to obtain analyses as representative as possible of the actual samples. Consequently, some methodologies have even been reported without the use of a solvent. ${ }^{2}$

Concerning synthetic polymers, we have witnessed oxidation side-products when analyzing dithiobenzoateterminated poly( $N$-acryloylmorpholine) chains (PNAM) synthesized by the Reversible Addition-Fragmentation chain Transfer (RAFT) controlled reversible deactivation radical polymerization process. ${ }^{5}$ Using as solvent THF, that is known to form peroxides, oxidation of the dithioester chain-end into sulfine and thioester was evidenced. Likewise, Zagorevskii et $a l^{6}$ have reported on the oxidation of the trithiocarbonate chain-end of polystyrene chains. They have notably detected sulfonic acid chain-ends while performing analyses on samples stored in THF for 1-3 days.

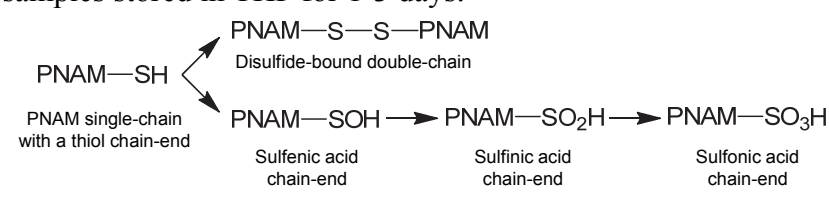

Scheme 1. Oxidation of the thiol chain-end of $\operatorname{poly}(N$ acryloylmorpholine), PNAM, during MALDI-TOF MS sample preparation may produce different PNAM chains-ends. 

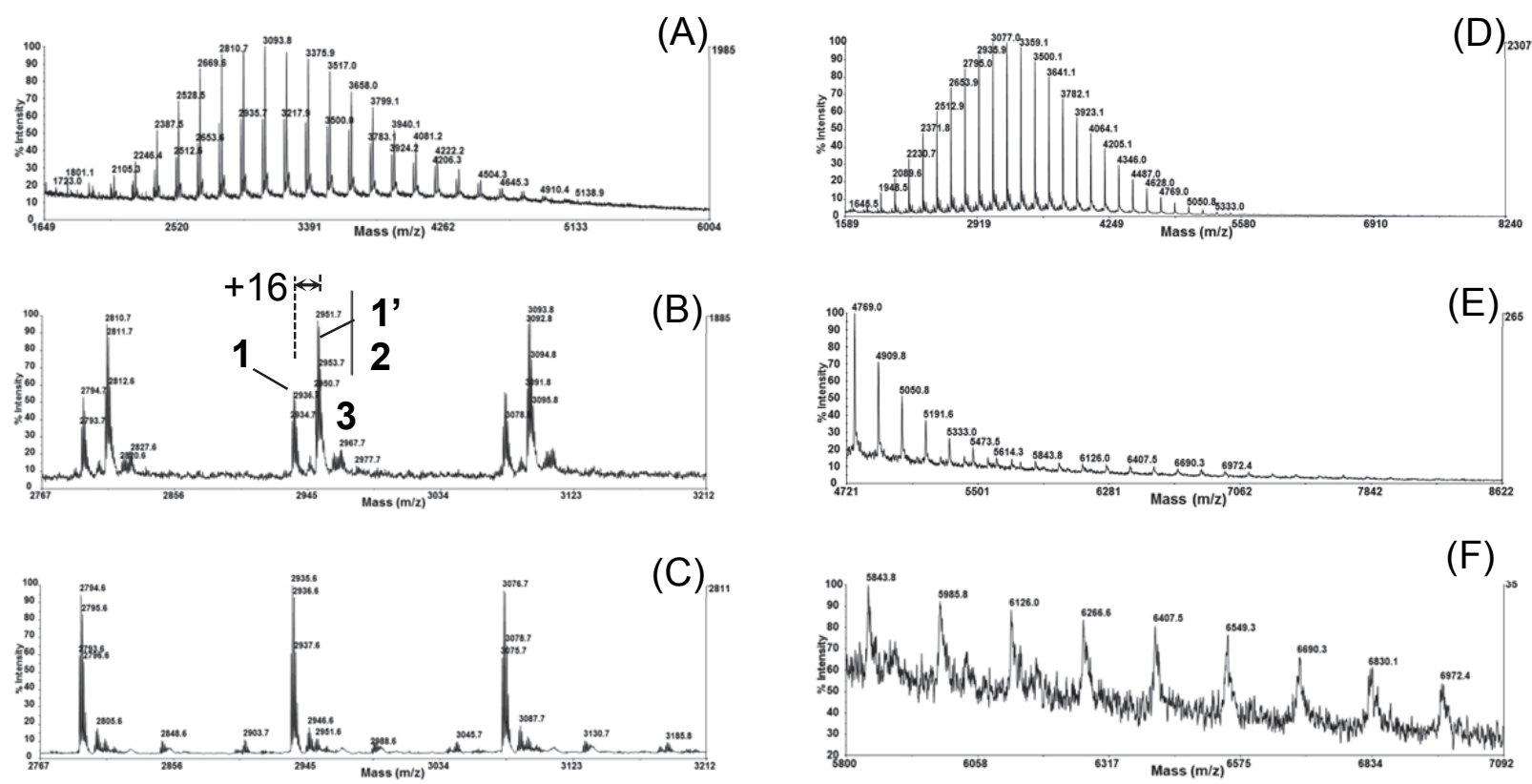

Figure 1. Repeatability of the positive MALDI-TOF mass spectrum of the same PNAM-SH sample (IAA matrix). Left) Reflectron mode analysis of the single-chain distribution (A) Full mass spectrum (B and C) enlargements of two different mass spectra. Right) Linear mode analysis showing two distributions of single- and double-chains. (D) Full mass spectrum, (E and F) enlargement of the double-chain distribution corresponding to disulfide-bound chains.

In the present work, we first investigated the oxidation that we observed also with thiol-terminated PNAM chains. A series of analyses on the same reference sample indicated the formation of different types of oxidized side-products (Scheme 1), in varying amounts according to the analysis. Second, considering this lack of reproducibility, we found that oxidation can actually be advantageously exploited in complementary analyses to more clearly identify the thiol chain-ends. Indeed, this methodology helped us to more extensively characterize polymer samples exhibiting various types of thiol-containing chain-ends.

\section{RESULTS AND DISCUSSION}

Variability of thiol chain-end oxidation during MALDITOF MS sample preparation. In the first part of the study, our aim was to assess the variability of thiol oxidation and the spectra using a reference polymer.

In previous research works, we have indeed obtained different results when analyzing the thiol chain-end of synthetic PNAM chains (PNAM-SH, obtained after aminolysis of the thiocarbonylthio- chain-end of the corresponding RAFT polymer). In some instances it has been nicely detected. ${ }^{7}$ However, in other cases, we have evidenced the formation of polymer chains with twice the expected molecular weight. ${ }^{5}$ MALDI-TOF MS analysis (positive-ions) indicated that these double-chains were linked by a disulfide bond resulting from the thiol chain-end oxidation (Scheme 1). In the latter case, data analysis is then impaired by a marked and poorly reproducible molar mass discrimination effect that tends to overestimate the single-chains. The detection sensitivity is indeed known to be higher for single- than for double-chains, ${ }^{8-9}$ resulting in mass spectra that may not be representative of the actual sample.
This illustrates the difficulty and the challenges related to the characterization of such thiol-containing polymers by MALDI-TOF MS. Oxidation may be influenced by many sample preparation parameters (solvent, temperature, drying time...) leading to variable amounts of different side-products and thus to poor analysis reproducibility.

To gain more insights, several independent analyses were conducted here with the same reference polymer sample for which the presence of a thiol chain-end was ascertained. For the sake of comparison, a low molecular weight PNAM-SH sample $\left(M_{\mathrm{n}}=3300 \mathrm{~g} \cdot \mathrm{mol}^{-1}, Ð=1.02\right.$, as determined by multiangle light scattering-size exclusion chromatography, MALSSEC) was prepared and used for all the analyses. It was synthesized by aminolysis of a corresponding RAFT polymer following our previously described procedure (see Supporting Information, SI). The latter procedure led quantitatively to the formation of a thiol chain-end, as investigated by several complementary techniques, MALDI-TOF MS, NMR and SEC coupled with MALS and UV-VIS absorption detectors (Figure $\mathrm{S} 1)^{5,} 7$ As an additional confirmation, we have further demonstrated that such thiol $\omega$-chain-end can be used for the quantitative introduction of an activated ester end-group by thiol-ene click chemistry with $N$-acryloxysuccinimide. ${ }^{10}$

Several independent MALDI-TOF MS analyses of the PNAM-SH sample highlighted the difficulty to obtain reproducible mass spectra. One spectrum exhibited a significant proportion of thiol chain-end oxidation. The mass spectrum exhibited a single-chain distribution ranging from ca. 1800 to $5000 \mathrm{~m} / \mathrm{z}$ (Figure S2) composed of several populations (Figure 1A). Enlargement of the reflectron mass spectrum (Figure 1B) revealed the repetition of peaks spaced by one NAM repetitive unit $(c a .141 \mathrm{~m} / \mathrm{z})$. 

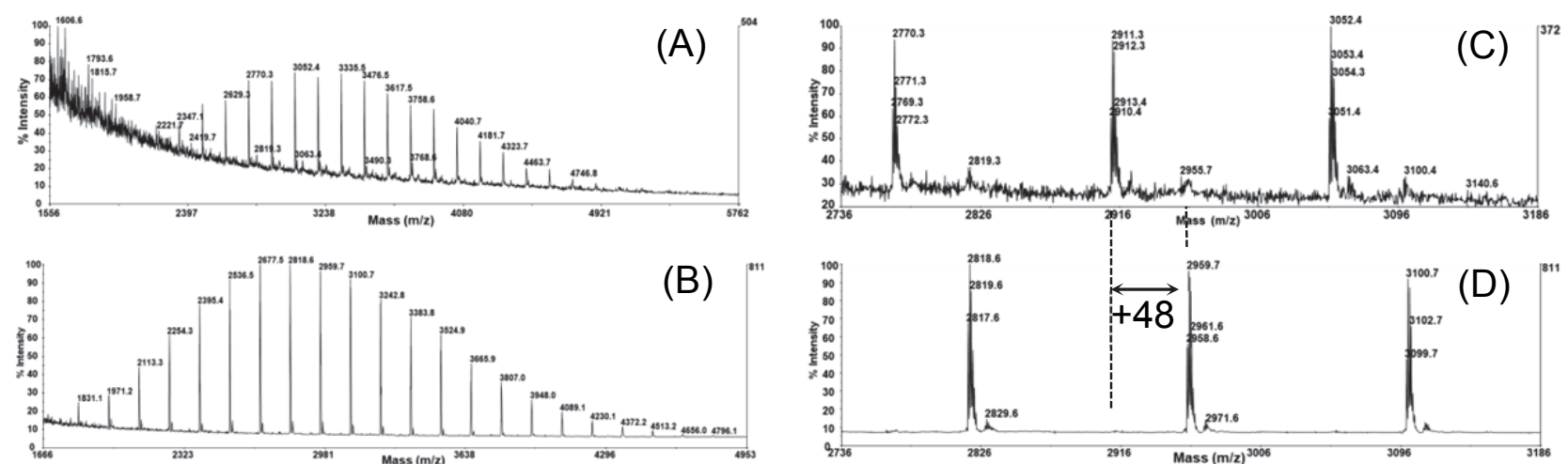

Figure 2. Negative-ion MALDI-TOF mass spectra of the same PNAM-SH sample in reflectron mode (IAA matrix). (A), (B) Full spectra, (C), (D) Corresponding enlargements centered on $\mathrm{DP}=20$. Analyses were performed using commercial (top panels) or distilled THF (bottom panels)

Three populations corresponding to three different types of chain-ends were detected. The expected thiol-terminated PNAM-SH chains were clearly observed with a $\mathrm{Na}^{+}$or $\mathrm{K}^{+}$ cationization (Population 1 and 1', e.g. for $\mathrm{DP}=20$, $\left[\mathrm{HS}\left(\mathrm{C}_{7} \mathrm{H}_{11} \mathrm{NO}_{2}\right)_{20} \mathrm{C}_{4} \mathrm{H}_{9}+\mathrm{Na}\right]^{+}=\mathrm{C}_{144} \mathrm{H}_{230} \mathrm{~N}_{20} \mathrm{O}_{40} \mathrm{~S}_{1} \mathrm{Na}_{1}$, calculated $2934.6 \mathrm{~m} / \mathrm{z}$, found $2934.7 \mathrm{~m} / \mathrm{z}$ ). Nonetheless, another main population with a similar proportion at $+16 \mathrm{~m} / \mathrm{z}$ (Population 2) was observed, superimposed to the previous $\mathrm{K}^{+}$ (Population 1'). The mass difference corresponding to an additional oxygen atom, this Population 2 was attributed to an oxidation product, tentatively PNAM-SOH with a sulfenic acid chain-end (Scheme 1). Moreover, there was a minor population (Population 3) at $+32 \mathrm{~m} / \mathrm{z}$ that was assigned to PNAM- $\mathrm{SO}_{2} \mathrm{H}$ chains (with a sulfinic acid chain-end, Scheme $1)$.

New analyses with the same PNAM-SH sample was repeated in more controlled conditions, i.e. THF solution was systematically prepared shortly before analysis. In these conditions, mass spectra significantly differed from the previous results. Solely the expected thiol-terminated PNAMSH chains were detected, either with an already- (Figure 1C) or freshly-opened (Figure S3) THF solvent bottle. No population at $+16 \mathrm{~m} / \mathrm{z}$ or at $+32 \mathrm{~m} / \mathrm{z}$ was observed. However, the mass spectra exhibited two distributions (Figure 1D-F): predominantly a single-chain distribution but also a doublechain distribution centered around $6400 \mathrm{~m} / \mathrm{z}$ with a much lower signal-to-noise ratio. Enlargements (Figure 1E-F) showed that this double-chain distribution corresponded to oxidized chains linked together by a disulfide bond (PNAM-S$\mathrm{S}-\mathrm{PNAM}$ in Scheme 1 , e.g. for $\mathrm{DP}=40$, $\left[\mathrm{C}_{4} \mathrm{H}_{9}\left(\mathrm{C}_{7} \mathrm{H}_{11} \mathrm{NO}_{2}\right)_{20} \mathrm{~S}_{2}\left(\mathrm{C}_{7} \mathrm{H}_{11} \mathrm{NO}_{2}\right)_{20} \mathrm{C}_{4} \mathrm{H}_{9}+\quad \mathrm{Na}\right]^{+}=$ $\mathrm{C}_{288} \mathrm{H}_{458} \mathrm{~N}_{40} \mathrm{O}_{80} \mathrm{~S}_{2}$, calculated average mass $5844.2 \mathrm{~m} / \mathrm{z}$, found $5843.8 \mathrm{~m} / \mathrm{z}$ ). Although the apparent lower proportion of this double-chain distribution, its actual proportion is questionable due to the above-mentioned mass discrimination effect.

In conclusion, independent MALDI-TOF MS analyses of the same sample show that chain-end oxidation can i) produce different kinds of oxidized products, and ii) be variable depending on sample preparation. Such an oxidation may be detrimental for data analysis. A great care should thus be taken when preparing such thiol-containing MALDI-TOF samples to obtain reproducible results and accurate chain-end analysis. Nevertheless, oxidation proved difficult to be fully avoided. Moreover, preparing samples just before analysis may be problematic, especially when dealing with a large number of samples. Therefore, we explored if complementary MALDI-
TOF MS analyses could help to more clearly evidence the presence of thiol chain-ends.

Complementary analyses to confirm thiol chain-end of polymer chains. Two approaches were investigated: i) detection of PNAM-SH chains in their deprotonated thiolate form, PNAM-S, by negative-ion analyses, and ii) use of THF without anti-oxidant stabilizer instead of commercial THF, as the solvent.

i) When performing negative-ion analysis of the same PNAM-SH sample in commercial THF (Figure 2A, 2C and SI4), no double-chain distribution was observed, as expected. The single-chain distribution was mainly composed of the thiolate-ended chains (PNAM-S, e.g. for $\mathrm{DP}=20$, $\left[\mathrm{C}_{144} \mathrm{H}_{230} \mathrm{~N}_{20} \mathrm{O}_{40} \mathrm{~S}-\mathrm{H}\right]^{-}$, calculated $2910.6 \mathrm{~m} / \mathrm{z}$, found 2910.4 $\mathrm{m} / \mathrm{z}$ ). Thus, despite the lower sensitivity than for the corresponding positive-ion analyses (Figure 1), detection of thiolate chain-ends in the negative-ion mode appears as an efficient complement to confirm the presence of the thiol chain-end.

ii) The use of THF devoid of anti-oxidant stabilizer (after distillation) altered the oxidation pattern during sample preparation, as shown by both positive-ion and negative-ion analyses (Figure 2B, 2D and S5). Positive-ion analyses presented a lower sensitivity of detection (low signal-to-noise ratio in the linear mode, Figure S5A), compared to the previous analyses with commercial THF (Figure 1). Linear mass spectrum exhibited both a single-chain and a doublechain distribution. The single-chain distribution was composed of two populations including the expected one and another at $+16 \mathrm{~m} / \mathrm{z}$, previously attributed to oxidized sulfenic acid chainends (Figure S5B). Furthermore, the double-chain distribution noticed on the high molar mass side of the spectrum corresponded to disulfide chains (Figure S5A).

More strikingly, the negative-ion mass spectrum was obtained with a very satisfactory sensitivity (Figure 2B and $\mathrm{S} 5 \mathrm{C}$ ), in contrast with the same analysis performed with commercial THF (Figure 2A). In addition, the unique singlechain distribution was composed of one major population. The latter was not PNAM-S $\mathrm{S}^{-}$chains with a thiolate chain-end (appearing as a minor population), but rather a $+48 \mathrm{~m} / \mathrm{z}$ one. This mass difference corresponding to three oxygen atoms, it could 


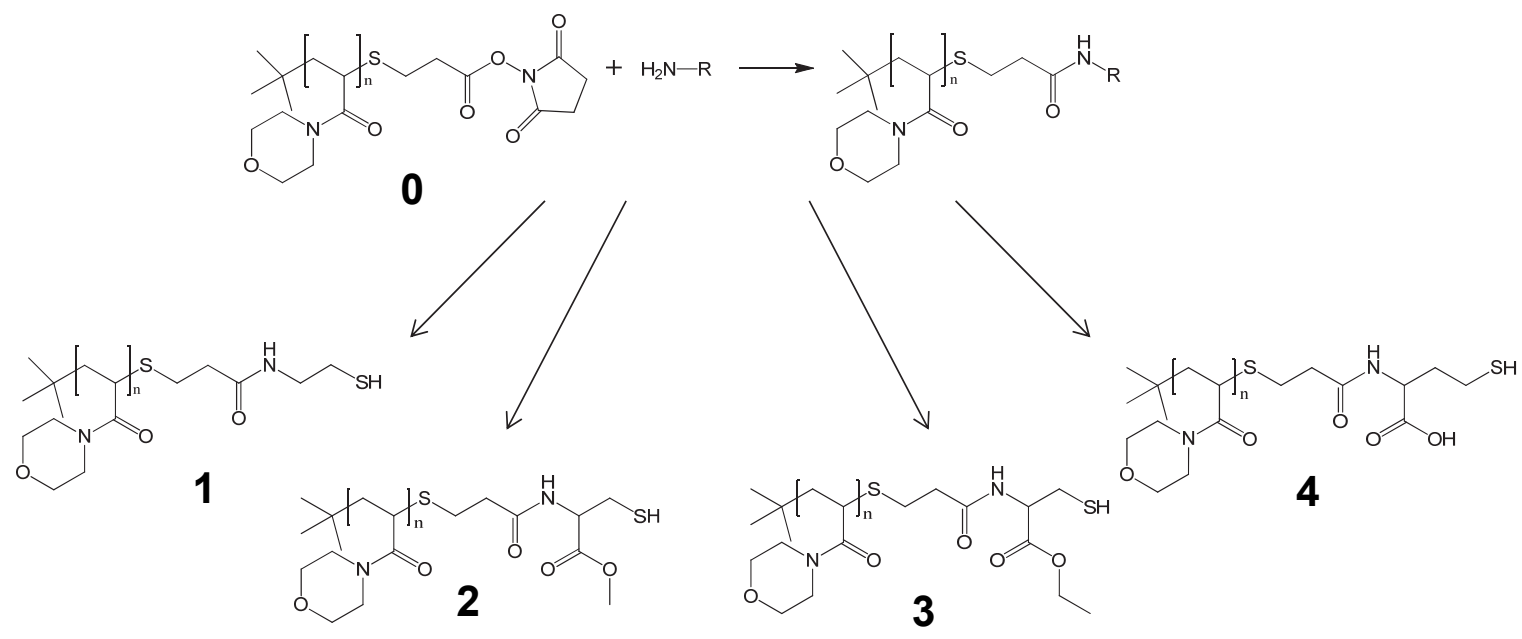

Scheme 2. Various thiol-terminated PNAM chains (polymers 1-5) prepared from PNAM chains bearing a succinimidyl ester (noted 0)
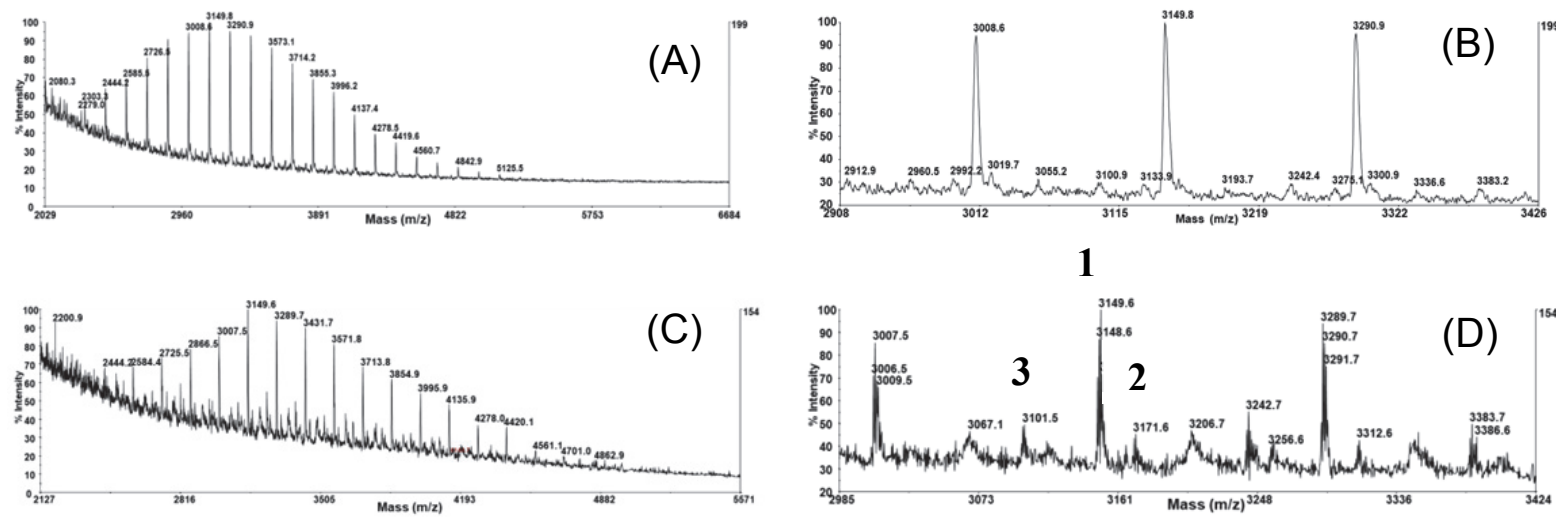

Figure 3. Negative-ion analysis of polymers 2 and 4 using distilled THF. (A) Full linear mode mass spectrum of polymer 2. (B) Enlargement of A. (C) Full reflectron mode mass spectrum of polymer 4. (D) Enlargement of C.

be attributed to $\mathrm{PNAM}-\mathrm{SO}_{3}{ }^{-}$chains with a sulfonate chainend (e.g. for $\mathrm{DP}=20,\left[\mathrm{C}_{144} \mathrm{H}_{230} \mathrm{~N}_{20} \mathrm{O}_{43} \mathrm{~S}-\mathrm{H}\right]^{-}$, calculated $2958.6 \mathrm{~m} / \mathrm{z}$, found $2958.3 \mathrm{~m} / \mathrm{z}$ ), which is a known oxidation product of thiols in the presence of peroxides (Scheme 1). ${ }^{11}$ The much lower pKa (and thus the higher propensity to be negatively charged) of sulfonic acids compared to thiols probably explains the higher sensitivity of detection. (Note that in the positive-ion mass spectrum, this population is detected as a sodium sulfonate population, [PNAM- $\mathrm{SO}_{3} \mathrm{Na}+$ $\mathrm{Na}]^{+}$, Figure S5B).

Therefore, these results suggested that oxidation was favored with non-stabilized THF, probably because of the absence of anti-oxidant compared to commercial THF (stabilized with ca. $250 \mathrm{ppm}$ of butylated hydroxytoluene, BHT). A significant oxidation can thus occur during sample preparation and air-drying at the surface of the MALDI target, due to the formation of peroxides. A simple assay with peroxide test strips (e.g. Quantofix $\left.{ }^{\circledR}\right)$ indeed showed a marked difference between non-stabilized and stabilized THF (Figure S6). Although no peroxides were initially detected in both cases, when the strip was allowed to dry at air for few minutes ( $\sim 5 \mathrm{~min}$ ), the blue coloration indicating the formation of peroxides progressively got more intense in the case of nonstabilized THF. Conversely, with commercial stabilized THF, no such coloration appeared.

In conclusion, using non-stabilized THF can be seen as a way to force the oxidation of thiol into sulfonic acid groups that are then more sensitively detected by negative-ion analyses. This method may thus be advantageously used to confirm the thiol functionalization of polymers.

Taking advantage of oxidation to identify various types of thiol chain-ends. As an illustration, we used this complementary method to characterize a series of PNAM chains bearing various types of thiol chain-ends (noted 1-4 in Scheme 2). These polymers were synthesized from the same PNAM precursor bearing a succinimidyl ester chain-end (noted $\mathbf{0}$ in Scheme 2) prepared following a previously described procedure. ${ }^{10}$ MALDI-TOF MS was then used to prove the chain-end modification, especially by conducting negative-ion analyses with non-stabilized THF.

From a general perspective, the detection sensitivity for the new thiol-terminated polymers was lower than for the PNAM$\mathrm{SH}$ sample, evidencing an influence of the chain-end. ${ }^{12}$ When 
performing regular analyses with commercial THF, some oxidation could not be avoided, complicating the characterization of the reaction product (not shown). In this case, it was thus relevant to use non-stabilized THF, to force oxidation of thiols into sulfonic acids and to facilitate chainend characterization by negative-ion analysis.

Whereas the positive-ion complex spectra brought first proofs of the formation of the expected thiol-terminated chains (see SI and Figure S7), negative-ion analyses provided a much clearer confirmation (Figure 3 and S8).

For samples 1-3 only one distribution of single-chains was detected. It was composed of a unique population corresponding to the expected chains with a sulfonate chainend (Polymer- $\mathrm{SO}_{3}{ }^{-}, \quad$ e.g. for sample 2, $\mathrm{DP}=20$, $\left[\mathrm{C}_{151} \mathrm{H}_{241} \mathrm{~N}_{21} \mathrm{O}_{46} \mathrm{~S}_{2}-\mathrm{H}\right]^{-}$, calculated average mass: $3149.8 \mathrm{~m} / \mathrm{z}$, found $3149.8 \mathrm{~m} / \mathrm{z}$ ), resulting from thiol oxidation (Figure 3A$\mathrm{B}$ and S8). No hydrolysis into carboxylate groups of the activated ester chain-end of the initial polymer precursor (noted $\mathbf{0}$ in Scheme 1) was detected. Therefore, such analyses gave an unambiguous proof of the formation of the thiolcontaining chain-end.

For sample 4, analysis was more complex because this thiol-terminated polymer also carries a carboxylic acid group that can be deprotonated $(\mathrm{pKa} \sim 4)$. Consequently, for negative-ion analysis (Figure 3C-D), the polymer chains could be anionized by the formation of carboxylate groups (-COO$\left.{ }^{-}\right)$, in addition to the above-described pathways. Interestingly, for sample 4, a double-chain distribution was observed not only in the positive-ion mass spectrum (Figure S7D) but also in the negative-ion one (Figure S8C). In the latter case, the doublechain distribution corresponded to disulfide double-chains bearing one $\mathrm{COO}^{-}$anionized group, and was composed of several populations because the other carboxylic group of the chain can be present either as $-\mathrm{COOH},-\mathrm{COONa}$ or less likely as $-\mathrm{COOK}$. For the same reason, the single-chain distribution was also composed of several populations of expected chains (Figure 3C-D). For instance, chains with a sulfonate endgroup were detected with both a $-\mathrm{COOH}$ (main population noted 1 in Figure 3D) or a $-\mathrm{COONa}$ (secondary population noted 2) group (e.g. for $\mathrm{DP}=20$, Polymer- $\mathrm{SO}_{3}{ }^{-} / \mathrm{COOH}$, $\left[\mathrm{C}_{151} \mathrm{H}_{241} \mathrm{~N}_{21} \mathrm{O}_{46} \mathrm{~S}_{2}-\mathrm{H}\right]^{-}$, calculated mass: $3147.7 \mathrm{~m} / \mathrm{z}$, found $3147.6 \mathrm{~m} / \mathrm{z}$; Polymer-SO ${ }_{3}{ }^{-} / \mathrm{COONa},\left[\mathrm{C}_{151} \mathrm{H}_{240} \mathrm{~N}_{21} \mathrm{NaO}_{46} \mathrm{~S}_{2}-\mathrm{H}\right]$ , calculated mass: $3169.6 \mathrm{~m} / \mathrm{z}$, found $3169.6 \mathrm{~m} / \mathrm{z}$ ). A secondary Polymer-S $/ \mathrm{COOH}$ population (noted 3 in Figure 3D) was also visible $\left(\left[\mathrm{C}_{151} \mathrm{H}_{241} \mathrm{~N}_{21} \mathrm{O}_{43} \mathrm{~S}_{2}-\mathrm{H}\right]\right.$, calculated mass: $3099.7 \mathrm{~m} / \mathrm{z}$, found $3099.5 \mathrm{~m} / \mathrm{z}$ ). Such analyses were essential to definitely prove the formation of the expected product.

Those examples thus demonstrated that forcing oxidation during sample preparation is a very valuable method for the characterization of various types of thiol-containing polymer chain-ends. The lower $\mathrm{pKa}$ of sulfonic groups $(\mathrm{pKa}<0)$ compared to thiol groups ( $\mathrm{pKa} \sim 13)$ enables the detection, in negative-ion analyses, of a higher amount of chains and thus leads to a better sensitivity (signal-to-noise ratio). We thus recommend performing such analyses in complement to regular ones.

\section{ASSOCIATED CONTENT}

\section{Supporting Information}

The Supporting Information is available free of charge on the ACS Publications website.

Experimental section, complementary characterizations of the reference PNAM-SH polymer, theoretical average and monoiso- topic masses of positive and negative ions of possible PNAM structures and complementary MALDI-TOF spectra of the polymers.

\section{AUTHOR INFORMATION}

\section{Corresponding Author}

* catherine.ladavière@univ-lyon1.fr

*arnaud.favier@univ-lyon1.fr

ORCID

Catherine Ladavière: 0000-0002-6039-7471

Arnaud Favier: 0000-0002-7482-7874

\section{Author Contributions}

The manuscript was written through contributions of all authors. All authors have given approval to the final version of the manuscript.

\section{ACKNOWLEDGMENT}

We thank Agnès Crépet (Laboratoire d'Ingénierie des Matériaux Polymères) and the Liquid Chromatography Platform (Institut de Chimie de Lyon) for their expertise and technical support in SEC/MALLS. L.F. acknowledges a Ph.D. grant from the French Ministry of Research and Education.

\section{REFERENCES}

1. Schaiberger, A. M.; Moss, J. A., Optimized Sample Preparation for MALDI Mass Spectrometry Analysis of Protected Synthetic Peptides. J. Am. Soc. Mass Spectrom. 2008, 19, 614-619.

2. Trimpin, S.; Deinzer, M. L., Solvent-free MALDI-MS for the Analysis of $\beta$-Amyloid Peptides via the Mini-Ball Mill Approach: Qualitative and Quantitative Advances. J. Am. Soc. Mass Spectrom. 2007, 18, 1533-1543.

3. Baez, N. O. D.; Reisz, J. A.; Furdui, C. M., Mass spectrometry in studies of protein thiol chemistry and signaling: opportunities and caveats. Free Radic. Biol. Med. 2015, 80, 191-211.

4. Montaudo, G.; Samperi, F.; Montaudo, M. S., Characterization of synthetic polymers by MALDI-MS. Prog. Polym. Sci. 2006, 31 (3), 277-357.

5. Favier, A.; Ladaviere, C.; Charreyre, M. T.; Pichot, C., MALDITOF MS investigation of the RAFT polymerization of a water-soluble acrylamide derivative. Macromolecules 2004, 37, 2026-2034.

6. Zagorevskii, D. V.; Nasrullah, M. J.; Raghunadh, V.; Benicewicz, B. C., Rapid Commun. Mass Spectrom. 2006, 20, 178.

7. Cepraga, C.; Favier, A.; Lerouge, F.; Alcouffe, P.; Chamignon, C.; Lanoe, P.-H.; Monnereau, C.; Marotte, S.; Ben Daoud, E.; Marvel, J.; Leverrier, Y.; Andraud, C.; Parola, S.; Charreyre, M.-T., Fluorescent gold nanoparticles with chain-end grafted RAFT copolymers: influence of the polymer molecular weight and type of chromophore. Polym. Chem. 2016, 7, 6812-6825.

8. Montaudo, G.; Montaudo, M. S.; Puglisi, C.; Samperi, F., Rapid Commun. Mass Spectrom. 1995, 9, 453.

9. Martin, K.; Spickermann, J.; Räder, H. J.; Müllen, K., Why Does Matrix-assisted Laser Desorption/Ionization Time-of-flight Mass Spectrometry Give Incorrect Results for Broad Polymer Distributions? Rapid Commun. Mass Spectrom. 1996, 10, 1471-1474.

10. Duret, D.; Haftek-Terreau, Z.; Carretier, M.; Berki, T.; Ladavière, C.; Monier, K.; Bouvet, P.; Marvel, J.; Leverrier, Y.; Charreyre, M. T.; Favier, A., Labeling of native proteins with fluorescent RAFT polymer probes: application to the detection of a cell surface protein using flow cytometry. Polym. Chem. 2018, 9 , 1857-1868.

11. Ballistreri, F. P.; Tomaselli, G. A.; Toscano, R. M., Selective and mild oxidation of thiols to sulfonic acids by hydrogen peroxide catalyzed by methyltrioxorhenium. Tetrahedron Lett. 2008, 49, 32913293.

12. Alicata, R.; Montaudo, G.; Puglisi, C.; Samperi, F., Influence of chain end groups on the matrix-assisted laser desorption/ionization spectra of polymer blends. Rapid Commun. Mass Spectrom. 2002, 16 , 248-260. 
Table of Content Graphic.

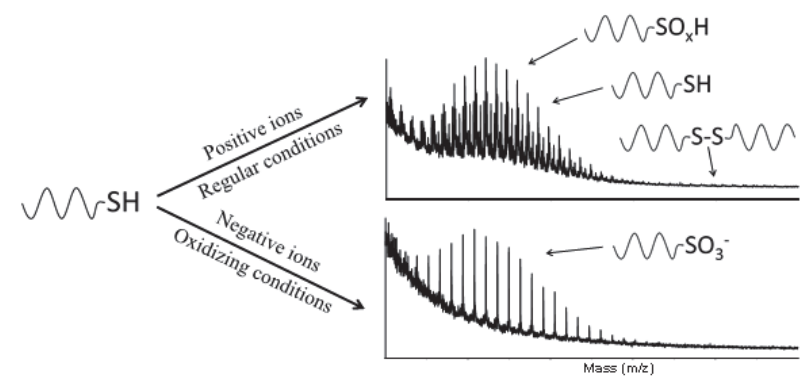

